\title{
Diet and risk of endometriosis in a population-based case-control study
}

\author{
Britton Trabert ${ }^{1,2,3 *}$, Ulrike Peters ${ }^{1,4}$, Anneclaire J. De Roos ${ }^{1,2}$, Delia Scholes ${ }^{5}$ and Victoria L. Holt ${ }^{1,2}$ \\ ${ }^{1}$ Department of Epidemiology, University of Washington, Seattle, WA 98195, USA \\ ${ }^{2}$ Epidemiology Research Unit, Fred Hutchinson Cancer Research Center, Seattle, WA 98109, USA \\ ${ }^{3}$ Division of Cancer Epidemiology and Genetics, National Cancer Institute, Rockville, MD 20852, USA \\ ${ }^{4}$ Cancer Prevention Program, Fred Hutchinson Cancer Research Center, Seattle, WA 98109, USA \\ ${ }^{5}$ Center for Health Studies, Group Health Cooperative, Seattle, WA 98101, USA \\ (Received 3 February 2010 - Revised 5 July 2010 - Accepted 20 August 2010 - First published online 28 September 2010)
}

\section{Abstract}

Diet plausibly has a role in the aetiology of endometriosis through effects on steroid hormone levels; however, few published studies have examined the diet and endometriosis risk. We evaluated dietary risk factors for endometriosis in a population-based case-control study. Cases were 284 Group Health (GH) enrollees aged 18-49 years with newly diagnosed, surgically confirmed endometriosis between 1996 and 2001. Controls were 660 randomly selected age-matched female GH enrollees without a history of endometriosis. Nutrients and selected food groups were assessed using the Women's Health Initiative FFQ. OR of endometriosis risk associated with dietary exposures were estimated using unconditional logistic regression and adjusted for identified covariates. Increased total fat consumption was associated with decreased endometriosis risk (fourth quartile $v$. lowest: OR $0 \cdot 5,95 \% \mathrm{CI} 0 \cdot 2,1 \cdot 0, P$-trend $=0 \cdot 12$ ). Increased $\beta$-carotene consumption and servings/d of fruit were associated with increased risk ( $\beta$-carotene third quartile $v$. lowest: OR 1·7, 95\% CI 1·1, 2.6; fourth quartile $v$. lowest: OR 1·6, $95 \%$ CI 1·0, 2·5, $P$-trend $0 \cdot 16$; fruit $>2$ servings $/ \mathrm{d} v$. $<1$ : OR $1 \cdot 5,95 \%$ CI $1 \cdot 0,2 \cdot 3, P$-trend=0.04). We also found a suggestion of decreased endometriosis risk associated with the consumption of dairy products $(2$ servings/d $v . \leq 1:$ OR $0 \cdot 6,>2$ servings/d $v . \leq 1$ : OR 0.7 ), but this association was not statistically significant for the highest tertile. The present study suggests that specific dietary components may be associated with endometriosis risk.

Key words: Case-control studies: Population-based studies: Diet: Endometriosis

Endometriosis is a gynaecological disorder characterised by the presence of functioning endometrial glands and stroma outside the uterus. The best population-based estimates of prevalence suggest that endometriosis affects $8-10 \%$ of reproductive-aged women in the United States ${ }^{(1)}$. Symptoms associated with endometriosis include severe menstrual pain, excessive menstrual bleeding, chronic and severe pelvic pain, dyspareunia and subfertility.

Increased exposure to oestrogens is a common link among several known risk factors for endometriosis. Local oestrogen production coupled with circulating oestrogen stimulates the proliferation of ectopic endometrial tissue potentially leading to endometriosis ${ }^{(2,3)}$. Menarche at early age, shorter menstrual cycle length, nulliparity and greater height are all risk factors for endometriosis that are also associated with increased levels of circulating oestrogen concentrations ${ }^{(4-8)}$.

The relationship between diet and endometriosis risk is not well characterised. Diet may have a role in the aetiology of endometriosis through its influence on steroid hormone levels, among other potential mechanisms. Some observational studies have shown that plant-based diets and diets high in fibre increase oestrogen excretion and decrease concentrations of bioavailable oestrogen, and thus may lower endometriosis $\operatorname{risk}^{(9,10)}$. Additionally, high-fat diets have been associated with increased serum oestrone, oestrone sulphate and oestradiol levels in premenopausal women ${ }^{(9,11)}$, suggesting that diets low in fat and high in fibre may modify endometriosis risk by altering steroid hormone metabolism.

The few published reports evaluating dietary risk factors for endometriosis have been somewhat contradictory. In the first of two case-control studies of endometriosis and specific foods published to date, increased consumption of ham, beef and red meat was associated with increased endometriosis risk in women visiting gynaecology clinics in northern Italy $^{(12)}$. Conversely, in that study, green vegetable, fresh fruit and oil were all associated with

Abbreviation: GH, Group Health.

*Corresponding author: B. Trabert, fax +1 301402 0916, email trabertbl@mail.nih.gov 
decreased risk ${ }^{(12)}$. In the second study, a Belgian clinicbased case-control study, butter was marginally associated with an increased risk of peritoneal endometriosis ${ }^{(13)}$. Recently, a large US cohort study evaluated dietary fat consumption and endometriosis risk using 12 years of prospectively collected data ${ }^{(14)}$. The authors of that study reported that total dietary fat intake was not associated with endometriosis risk, but increased long-chain n-3 fatty acid consumption was associated with decreased risk of endometriosis and trans-fat intake was associated with increased risk of endometriosis ${ }^{(14)}$. The purpose of this analysis was to further investigate the role of diet assessing both nutrients and a wide variety of food groups - as a risk factor for endometriosis in a population-based case-control study of US women.

\section{Materials and methods}

\section{Study population}

The present study was conducted within the Women's Risk of Endometriosis study, the details of which have been described elsewhere ${ }^{(15)}$. In brief, Women's Risk of Endometriosis was a population-based case-control study of reproductive-age, premenopausal women enrolled in Group Health $(\mathrm{GH})$ Cooperative, a large mixed-model healthcare organisation in Washington State. The cases were 18-49-year-old female GH enrollees with an incident endometriosis diagnosis (International Classification of Disease 9th Revision diagnostic codes 617·0-617.5, 617.8 and 617.9; individuals with International Classification of Disease 9th Revision code 617.0, uterine endometriosis, who did not meet the disease definition of endometriosis were excluded) between 1 April 1996 and 31 March 2001. Inpatient and outpatient medical record reviews confirmed the cases' lesion characteristics and diagnostic treatment details and provided their reference date (the date of the first GH visit for symptoms leading to the endometriosis diagnosis). Population-based controls were randomly selected from 18-49-year-old GH female enrollees without an endometriosis diagnosis during the study period; they were frequency matched on 5-year age intervals and assigned a reference date to correspond with the distribution of cases' reference dates. Women who did not speak English or who reported a hysterectomy or bilateral oophorectomy upon initial telephone eligibility screening were excluded from the participation. The present study was conducted according to the guidelines established in the Declaration of Helsinki, and all procedures involving human subjects were approved by the GH Institutional Review Board. A written informed consent was obtained from all the subjects. All the participants were interviewed using a structured questionnaire with questions regarding demographics, employment, prior medical conditions, menstrual history, pregnancy history, contraceptive methods, hormone use, tobacco and alcohol use, and family and personal history of endometriosis. A total of 340 cases ( $73 \%$ of those eligible) and 741 controls $(73 \%$ of those eligible) completed the interview on endometriosis risk factors. As a result of information captured on the interview, twelve cases and fourteen controls with a selfreported history of surgically confirmed endometriosis who were interviewed were excluded. While International Classification of Disease 9th Revision codes denoting any amount of endometriosis were the basis for initial case group selection, for the analyses presented here, we utilised a published case definition that emphasised definite or probable endometriotic disease, that is, endometriosis causing structural or functional damage or substantial symptoms ${ }^{(16)}$. As defined by Holt \& Weiss ${ }^{(16)}$, 'definite endometriotic disease' included ovarian endometriomas, pelvic endometriotic lesions over $5 \mathrm{~mm}$ deep and pelvic endometriotic lesions with adhesions not attributable to other causes. 'Probable endometriotic disease' included other endometriotic implants with at least one major endometriosis symptom (infertility, moderate or severe dysmenorrhoeal, dyspareunia or pelvic pain) ${ }^{(16)}$. As a result, cases without surgical evidence of disease and asymptomatic cases with superficial or ambiguous pelvic lesions were excluded ( $n$ 12), as were three cases of extrapelvic scar endometriosis. After these exclusions, 313 cases and 727 controls remained.

Cases (93\%, $n$ 291) and controls (93.1\%, $n$ 677) who participated in the main study interview and satisfied the case and control definitions outlined above also completed a self-administered Women's Health Initiative FFQ about their usual diet during the year preceding the interview ${ }^{(17)}$. Using the FFQ, participants classified their consumption of specific food items into predefined frequency categories, ranging from never or less than once a month to two or more times a day, with corresponding serving size categories (small, medium and large). Summary questions were asked about usual overall intake of fruits and vegetables $^{(18)}$. As a supplement to the Women's Health Initiative FFQ, women were asked to complete a brief questionnaire (thirty-five line-items and one summary question) about their usual frequency of fish and shellfish consumption.

Average daily intakes for twenty-seven nutrients were calculated from the FFQ using the University of Minnesota Nutrient Data System for Research database of food composition (Minneapolis, MN, USA) ${ }^{(19)}$. Questions related to food purchasing and preparation (e.g. fat added at table and while cooking) were used to adjust the calculations of nutrient content in specific line items. Vitamin and mineral intakes were determined from dietary sources as well as from dietary combined with supplementation sources. The latter showed associations with endometriosis similar to the former; therefore, we present findings for vitamins and minerals based on only dietary sources.

Selected food groups were created by calculating average daily intake for each line item of the FFQ 
using predefined frequency and portion categories and summing across line items to create the following categories: dairy, vegetable, fruit (excluding fruit juice), whole grains, legumes, red meat, poultry, fatty fish, nonfatty fish and seafood.

Approximately, $2 \cdot 4 \%$ of cases (7/291) and $2 \cdot 7 \%$ of controls $(17 / 677)$ were excluded because their reported total energy intake was $<2092$ or $>20920 \mathrm{~kJ} ; 284$ cases and 660 controls were used for the present analyses.

\section{Statistical analysis}

Unconditional logistic regression models, using the standard multivariate method to control for energy consumption $^{(20)}$, were used to estimate OR and $95 \%$ CI for the association between dietary factors and endometriosis, separately for nutrients and for food groups. Nutrient variables were included in the models as categorical variables, using quartiles based on the controls' distribution. To assess the linear trend in the log odds of nutrient quartile, the median value for each quartile was entered into the logistic regression model as an ordinal variable. Food group variables were included as servings/d or servings/ week. To assess the linear trend in the log odds of food group serving, the ordinal variable was entered into the logistic regression model.

All the analyses were adjusted for matching variables age-group and reference year, total energy intake as a continuous variable and confounding factors such as income, BMI, smoking status and alcohol consumption. BMI was included as an a priori confounding factor because of its known associations with dietary patterns and endometriosis ${ }^{(5,21)}$. Other covariates considered in the models included race, education, first-degree family history of endometriosis, physical activity, age at menarche, parity, history of breastfeeding and marijuana use. The addition of these covariates to a model controlling for age, reference year and BMI did not result in a $10 \%$ change in the $\beta$ coefficient of at least one quartile of total dietary fat, thus they were not included in the statistical analyses presented.

Endometriosis, as defined in the present study, included ovarian and non-ovarian cases and may be considered a heterogeneous disease entity. Therefore, in one subanalysis, we evaluated the association between dietary factors and ovarian and non-ovarian pelvic endometriosis separately. Since women who seek treatment for infertility may have endometriosis discovered incidentally as part of the diagnostic process, rather than because of symptomatic disease, we also considered separately the cases who reported seeking care only for reasons other than infertility. Finally, because of the possibility that there may be undiagnosed symptomatic cases in our population-based control group, we also conducted a subanalysis comparing endometriosis cases only with asymptomatic controls.

\section{Results}

The distributions of selected demographic and health characteristics for women who completed a dietary questionnaire are provided in Table 1. Cases and controls were similar with regard to age and race. A higher percentage of controls than cases reported household incomes of greater than $\$ 70000$, while a higher percentage of cases than controls smoked and drank alcohol.

Cases consumed slightly less energy than controls and had lower median intakes of total fat, cholesterol and $\mathrm{Ca}$ (Table 2). In multivariable adjusted analyses, increased total fat consumption was associated with a decreased endometriosis risk (fourth quartile $v$. lowest: OR 0.5, 95\% CI $0 \cdot 2, \quad 1 \cdot 0, \quad P$-trend $0 \cdot 12$ ) (Table 3). We observed similar inverse associations with endometriosis risk for saturated-, monounsaturated- and trans-fat intake.

Table 1. Demographic and health characteristics of the study population stratified by case-control status*

(Number and percentage values)

\begin{tabular}{|c|c|c|c|c|}
\hline \multirow[b]{2}{*}{ Characteristics } & \multicolumn{2}{|c|}{ Cases ( $n$ 284) } & \multicolumn{2}{|c|}{$\begin{array}{l}\text { Controls } \\
(n 660)\end{array}$} \\
\hline & $n \dagger$ & $\%$ & $n \dagger$ & $\%$ \\
\hline \multicolumn{5}{|l|}{ Age (years) } \\
\hline $18-24$ & 23 & $8 \cdot 1$ & 58 & 8.8 \\
\hline $25-34$ & 55 & $19 \cdot 4$ & 125 & 18.9 \\
\hline $35-44$ & 138 & $48 \cdot 6$ & 329 & 49.9 \\
\hline $45-49$ & 68 & 23.9 & 148 & $22 \cdot 4$ \\
\hline \multicolumn{5}{|l|}{ Race } \\
\hline Caucasian & 230 & $81 \cdot 0$ & 547 & 82.9 \\
\hline African American & 9 & 3.2 & 29 & 4.4 \\
\hline Asian American & 19 & $6 \cdot 7$ & 41 & $6 \cdot 2$ \\
\hline Other & 25 & 8.8 & 41 & $6 \cdot 2$ \\
\hline \multicolumn{5}{|l|}{ Income (\$) } \\
\hline$<35000$ & 83 & $29 \cdot 2$ & 180 & $27 \cdot 3$ \\
\hline $35000-49999$ & 60 & $21 \cdot 1$ & 145 & $22 \cdot 0$ \\
\hline $50000-69999$ & 71 & $25 \cdot 0$ & 134 & $20 \cdot 3$ \\
\hline $70000+$ & 60 & $21 \cdot 2$ & 183 & $27 \cdot 8$ \\
\hline \multicolumn{5}{|l|}{ Education (years) } \\
\hline$<12$ & 6 & $2 \cdot 1$ & 21 & $3 \cdot 2$ \\
\hline 12 & 47 & $16 \cdot 6$ & 119 & $18 \cdot 0$ \\
\hline$>12$ & 231 & $81 \cdot 3$ & 520 & $78 \cdot 8$ \\
\hline \multicolumn{5}{|l|}{$\operatorname{BMI}\left(\mathrm{kg} / \mathrm{m}^{2}\right)$} \\
\hline Underweight $(<18.5)$ & 7 & $2 \cdot 5$ & 13 & $2 \cdot 0$ \\
\hline Normal (18.5-24.9) & 144 & $50 \cdot 7$ & 345 & $52 \cdot 3$ \\
\hline Overweight (25-29.9) & 72 & $25 \cdot 4$ & 164 & 24.9 \\
\hline Obese $(>30)$ & 60 & $21 \cdot 1$ & 133 & $20 \cdot 2$ \\
\hline \multicolumn{5}{|l|}{ Smoking } \\
\hline Present & 55 & $19 \cdot 4$ & 103 & $15 \cdot 6$ \\
\hline Former & 66 & 23.2 & 162 & $24 \cdot 6$ \\
\hline Never & 163 & $57 \cdot 4$ & 395 & 59.9 \\
\hline \multicolumn{5}{|l|}{ Alcohol use } \\
\hline Present & 154 & $54 \cdot 2$ & 292 & $44 \cdot 2$ \\
\hline Former & 51 & $18 \cdot 0$ & 140 & $21 \cdot 2$ \\
\hline Never & 78 & 27.5 & 228 & 34.6 \\
\hline \multicolumn{5}{|l|}{ Parity (any live births) } \\
\hline Parous & 148 & $52 \cdot 1$ & 467 & $70 \cdot 8$ \\
\hline Nulliparous & 136 & 47.9 & 192 & $29 \cdot 1$ \\
\hline \multicolumn{5}{|l|}{ History of infertility } \\
\hline Yes & 66 & 23.2 & 86 & $13 \cdot 0$ \\
\hline No & 218 & $76 \cdot 8$ & 574 & 87.0 \\
\hline
\end{tabular}

${ }^{*}$ Restricted to participants who reported consuming between 2092 and $20920 \mathrm{~kJ} / \mathrm{d}$. † Cell counts may not sum to column total because of missing data. 
Table 2. Median daily intake of nutrients stratified by case and control status

\begin{tabular}{|c|c|c|}
\hline Nutrients & Cases ( $n$ 284) & Controls ( $n 660)$ \\
\hline Total energy (kJ) & 6237.50 & 6378.93 \\
\hline Protein $(\mathrm{g})$ & 64.4 & $65 \cdot 2$ \\
\hline Animal protein (g) & $45 \cdot 4$ & $45 \cdot 2$ \\
\hline Vegetable protein (g) & $17 \cdot 7$ & $18 \cdot 9$ \\
\hline Carbohydrates (g) & $178 \cdot 8$ & $186 \cdot 0$ \\
\hline Total fat $(\mathrm{g})$ & $54 \cdot 8$ & $58 \cdot 3$ \\
\hline Saturated fat $(\mathrm{g})$ & $19 \cdot 2$ & $21 \cdot 1$ \\
\hline Monounsaturated fat $(\mathrm{g})$ & $20 \cdot 3$ & 21.7 \\
\hline Polyunsaturated fat (g) & $11 \cdot 1$ & $11 \cdot 2$ \\
\hline Trans-fat $(\mathrm{g})$ & 3.2 & 3.4 \\
\hline Cholesterol (mg) & $190 \cdot 9$ & $197 \cdot 6$ \\
\hline$n-3$ Fatty acids $(\mathrm{g})$ & $1 \cdot 2$ & $1 \cdot 2$ \\
\hline$n-6$ Fatty acids (g) & $9 \cdot 8$ & 9.9 \\
\hline Fibre (g) & 13.9 & $14 \cdot 7$ \\
\hline Total folate $(\mu \mathrm{g})$ & $311 \cdot 0$ & $316 \cdot 8$ \\
\hline$\beta$-Carotene $(\mu \mathrm{g})$ & $1941 \cdot 2$ & 1878.5 \\
\hline Vitamin A - retinol $(\mu \mathrm{g})$ & $366 \cdot 1$ & 359.4 \\
\hline Vitamin C (mg) & $69 \cdot 2$ & $66 \cdot 8$ \\
\hline Vitamin D $(\mu \mathrm{g})$ & 3.7 & 3.7 \\
\hline Vitamin $\mathrm{E}(\mathrm{mg})$ & $6 \cdot 7$ & $7 \cdot 1$ \\
\hline Vitamin $B_{6}(\mathrm{mg})$ & 1.4 & 1.4 \\
\hline Vitamin $B_{12}(\mu \mathrm{g})$ & 5.4 & $5 \cdot 0$ \\
\hline Thiamin (mg) & $1 \cdot 2$ & $1 \cdot 2$ \\
\hline Riboflavin (mg) & 1.8 & 1.8 \\
\hline Niacin (mg) & $15 \cdot 9$ & $15 \cdot 7$ \\
\hline $\mathrm{Ca}(\mathrm{mg})$ & 754.4 & $796 \cdot 2$ \\
\hline $\mathrm{Fe}(\mathrm{mg})$ & $10 \cdot 6$ & $10 \cdot 9$ \\
\hline
\end{tabular}

We further evaluated the dietary fat association using adjustment questions from the Women's Health Initiative FFQ and found that women who reported primarily using butter, shortening or margarine while cooking and at the table were also at a decreased risk of endometriosis (butter, shortening or margarine for cooking $v$. no-fat added while cooking: OR 0.4, $95 \% \mathrm{CI} 0 \cdot 2,0 \cdot 8$; butter, shortening or margarine $v$. no-fat added at the table: OR $0 \cdot 5$, $95 \%$ CI $0 \cdot 2,1 \cdot 0)$. Increased $\beta$-carotene consumption was associated with increased endometriosis risk (third quartile $v$. lowest: OR $1 \cdot 7,95 \%$ CI 1·1, 2.6; fourth quartile $v$. lowest: OR 1.6, 95\% 1·0, 2.5, P-trend 0.16). Other macronutrients and micronutrients were not significantly associated with endometriosis risk.

We also evaluated daily or weekly servings of selected food groups as predictors of endometriosis risk (Table 4). Increased number of servings/d of fruit was associated with increased disease risk $(>1-2$ servings/d $v . \leq 1$ : OR $1 \cdot 1,95 \%$ CI $0 \cdot 8,1 \cdot 6 ;>2$ servings/d $v$. $\leq 1$ : OR $1 \cdot 5,95 \% \mathrm{CI}$ $1 \cdot 0,2 \cdot 3, P$-trend $0 \cdot 04$ ). Analyses exploring the effect of specific types of fruit did not identify any one type that primarily accounted for the increased endometriosis risk. We also found a suggestion of decreased endometriosis risk associated with consumption of dairy products ( 2 servings/ $\mathrm{d} v . \leq 1$ : OR $0 \cdot 6 ;>2$ servings/d $v . \leq 1$ : OR $0 \cdot 7)$, but this association was not statistically significant for the highest tertile. We did not observe significant associations with endometriosis risk for the other food groups evaluated.

Restricting analyses to cases with ovarian endometriosis only, the individual quartile associations between endometriosis and the following dietary components, dietary fat, $\beta$-carotene, dairy and fruit, did not diminish (results not shown). Compared with the results in Tables 3 and 4, the OR did not substantially change in analyses restricted to asymptomatic controls, suggesting that the presence of undiagnosed symptomatic cases in our control group was unlikely to have affected the present study (results not shown)

\section{Discussion}

Within the Women's Risk of Endometriosis study population, we observed decreased endometriosis risk with increased intakes of total fat and dairy. Our findings also suggested that higher intake of $\beta$-carotene and fruit were associated with an increased risk of endometriosis. Our data were not supportive of a relationship between endometriosis and any of the other nutrients or food groups examined.

Previous case-control studies exploring diet and risk of endometriosis were limited and have yielded inconsistent results. One report combined data from two case-control studies of endometriosis in northern Italy ${ }^{(12)}$. Cases ( $n$ 504) were women with laparoscopically confirmed endometriosis selected from obstetric and gynaecology clinics and controls ( $n$ 504) were women admitted to the same clinics for acute non-gynaecological, non-hormonal, non-neoplastic conditions. Dietary assessment was via interview; women were asked to report the number of portions/week of selected food items (major sources of retinoids and carotenoids in the Italian diet, alcohol and coffee consumption) in the year before the interview. Fat intake and consumption of whole grain foods were estimated by respondents as low, intermediate or high in quantity.

The second was a clinic-based case-control study of risk factors for endometriosis in Belgium ${ }^{(13)}$. Cases were eightyeight women diagnosed with peritoneal endometriosis and hospitalised for surgical treatment between January 2001 and December 2003; eighty-eight matched controls were recruited during the same time period from the same gynaecologists who referred the cases and had no clinical suspicion of peritoneal endometriosis. Consumption of selected foods was assessed with an FFQ; however, results of the present study were based on crude consumption frequencies, and nutrient consumption based on the FFQ was not reported.

The differences between the two case-control studies and our population-based study in subject selection criteria, dietary assessment and type of analysis (selected food groups $v$. nutrients) are substantial and limit our ability to compare the results. Both of the previous casecontrol studies reported components of dietary fat (butter, margarine and/or oil) rather than total fat based on nutrient values from an FFQ as in the present study. The Italian study reported a decreased risk of 
Table 3. Endometriosis and dietary nutrients among 284 cases and 660 controls

(Odds ratios and $95 \%$ confidence intervals)

\begin{tabular}{|c|c|c|c|c|c|c|c|c|}
\hline & \multirow[b]{2}{*}{ Reference } & \multicolumn{2}{|c|}{ Quartile 2} & \multicolumn{2}{|c|}{ Quartile 3} & \multicolumn{2}{|c|}{ Quartile 4} & \multirow[b]{2}{*}{$P$-trend } \\
\hline & & OR & $95 \% \mathrm{Cl}$ & OR & $95 \% \mathrm{Cl}$ & OR & $95 \% \mathrm{Cl}$ & \\
\hline \multicolumn{9}{|l|}{ Protein } \\
\hline Median (g) & 37.78 & $55 \cdot 68$ & & 72.86 & & 102.89 & & \\
\hline Case/control & $76 / 161$ & $73 / 168$ & & $61 / 161$ & & $74 / 170$ & & \\
\hline Multivariable adjusted ${ }^{*}$ & 1.0 & 1.0 & $0.6,1 \cdot 7$ & 0.9 & $0.5,1 \cdot 7$ & 1.2 & $0 \cdot 6,2 \cdot 6$ & 0.54 \\
\hline \multicolumn{9}{|l|}{ Protein (animal) } \\
\hline Median $(\mathrm{g})$ & $22 \cdot 3$ & 37.93 & & $52 \cdot 28$ & & 75.58 & & \\
\hline Case/control & $76 / 164$ & $65 / 165$ & & $76 / 172$ & & $67 / 159$ & & \\
\hline Multivariable adjusted ${ }^{\star}$ & 1.0 & 0.8 & $0.5,1.3$ & $1 \cdot 1$ & $0.7,1.8$ & $1 \cdot 1$ & $0.6,2.0$ & 0.61 \\
\hline \multicolumn{9}{|l|}{ Protein (vegetable) } \\
\hline Median $(\mathrm{g})$ & $10 \cdot 81$ & $16 \cdot 56$ & & 21.46 & & 33.21 & & \\
\hline Case/control & $84 / 173$ & $76 / 159$ & & $63 / 164$ & & $61 / 164$ & & \\
\hline Multivariable adjusted ${ }^{*}$ & 1.0 & 1.0 & $0.7,1.6$ & 0.8 & $0.5,1.3$ & 0.8 & $0.4,1.5$ & 0.40 \\
\hline \multicolumn{9}{|l|}{ Carbohydrate } \\
\hline Median (g) & $104 \cdot 70$ & $160 \cdot 42$ & & 209.44 & & 296.90 & & \\
\hline Case/control & $72 / 165$ & $82 / 165$ & & $62 / 165$ & & $68 / 165$ & & \\
\hline Multivariable adjusted* & 1.0 & 1.3 & $0.8,2 \cdot 2$ & 1.1 & $0.6,2 \cdot 0$ & 1.3 & $0 \cdot 6,2 \cdot 9$ & 0.70 \\
\hline \multicolumn{9}{|l|}{ Fat } \\
\hline Median (g) & $31 \cdot 78$ & 49.44 & & 67.74 & & $100 \cdot 17$ & & \\
\hline Case/control & $90 / 166$ & $66 / 159$ & & $63 / 167$ & & $65 / 168$ & & \\
\hline Multivariable adjusted ${ }^{\star}$ & 1.0 & $0.6 \dagger$ & $0.4,1.0$ & $0.6 \dagger$ & $0.3,1.0$ & 0.5 & $0.2,1.0$ & 0.12 \\
\hline Saturated fat & & & & & & & & \\
\hline Median (g) & 7.85 & 15.40 & & 24.53 & & 37.39 & & \\
\hline Case/control & $34 / 64$ & $123 / 265$ & & $66 / 168$ & & $61 / 163$ & & \\
\hline Multivariable adjusted ${ }^{\star}$ & 1.0 & 0.7 & $0.4,1 \cdot 1$ & 0.5 & $0 \cdot 3,1 \cdot 1$ & 0.5 & $0 \cdot 2,1 \cdot 1$ & 0.13 \\
\hline Monounsaturated fat & & & & & & & & \\
\hline Median (g) & $11 \cdot 32$ & $18 \cdot 38$ & & $25 \cdot 75$ & & 38.39 & & \\
\hline Case/control & $82 / 149$ & $76 / 184$ & & $56 / 154$ & & $70 / 173$ & & \\
\hline Multivariable adjusted ${ }^{\star}$ & 1.0 & 0.7 & $0.4,1 \cdot 0$ & $0.5 \dagger$ & $0.3,0.9$ & 0.6 & $0.3,1 \cdot 2$ & 0.25 \\
\hline Polyunsaturated fat & & & & & & & & \\
\hline Median $(\mathrm{g})$ & 6.05 & $9 \cdot 40$ & & $13 \cdot 25$ & & $20 \cdot 48$ & & \\
\hline Case/control & $81 / 176$ & $59 / 145$ & & $84 / 180$ & & $60 / 159$ & & \\
\hline Multivariable adjusted* & 1.0 & 0.9 & $0.6,1.4$ & $1 \cdot 0$ & $0.6,1 \cdot 6$ & 0.8 & $0.4,1.6$ & 0.65 \\
\hline Trans-fat & & & & & & & & \\
\hline Median $(\mathrm{g})$ & 1.60 & $2 \cdot 70$ & & $4 \cdot 16$ & & 7.39 & & \\
\hline Case/control & $95 / 171$ & $55 / 155$ & & $73 / 169$ & & $61 / 165$ & & \\
\hline Multivariable adjusted ${ }^{\star}$ & 1.0 & $0.6 \dagger$ & $0.4,1 \cdot 0$ & 0.7 & $0.4,1 \cdot 1$ & 0.6 & $0.3,1.1$ & 0.22 \\
\hline Cholesterol & & & & & & & & \\
\hline Median (mg) & $103 \cdot 16$ & 171.55 & & 237.53 & & 383.71 & & \\
\hline Case/control & $81 / 163$ & $67 / 168$ & & $73 / 164$ & & $63 / 165$ & & \\
\hline Multivariable adjusted ${ }^{\star}$ & 1.0 & 0.8 & $0.5,1 \cdot 2$ & 0.9 & $0.5,1.4$ & 0.7 & $0.4,1 \cdot 2$ & 0.33 \\
\hline$n-3$ Fatty acids & & & & & & & & \\
\hline Median (g) & 0.68 & 1.05 & & 1.42 & & $2 \cdot 11$ & & \\
\hline Case/control & $81 / 187$ & $62 / 124$ & & $80 / 183$ & & $61 / 166$ & & \\
\hline Multivariable adjusted ${ }^{*}$ & 1.0 & $1 \cdot 1$ & $0.7,1.7$ & 0.9 & $0.6,1.5$ & 0.7 & $0.4,1.4$ & 0.24 \\
\hline$n-6$ Fatty acids & & & & & & & & \\
\hline Median (g) & $5 \cdot 27$ & 8.29 & & 11.77 & & 17.94 & & \\
\hline Case/control & $76 / 177$ & $69 / 155$ & & $73 / 161$ & & $66 / 167$ & & \\
\hline Multivariable adjusted ${ }^{\star}$ & 1.0 & $1 \cdot 1$ & $0.7,1 \cdot 7$ & $1 \cdot 1$ & $0.6,1.8$ & 1.0 & $0.5,2.0$ & 0.99 \\
\hline Fibre & & & & & & & & \\
\hline Median (g) & 8.03 & $12 \cdot 48$ & & $16 \cdot 82$ & & $24 \cdot 26$ & & \\
\hline Case/control & $82 / 161$ & $74 / 169$ & & $55 / 167$ & & $73 / 163$ & & \\
\hline Multivariable adjusted* & 1.0 & 0.9 & $0.6,1.4$ & 0.8 & $0.5,1 \cdot 2$ & 1.0 & $0.6,1.8$ & 0.86 \\
\hline Folate & & & & & & & & \\
\hline Median $(\mu \mathrm{g})$ & 184.04 & 278.74 & & 356.82 & & $522 \cdot 30$ & & \\
\hline Case/control & $85 / 165$ & $61 / 165$ & & $68 / 164$ & & 70/166 & & \\
\hline Multivariable adjusted* & $1 \cdot 0$ & 0.7 & $0.5,1 \cdot 2$ & 0.8 & $0.5,1.4$ & 0.9 & $0.5,1.5$ & 0.87 \\
\hline$\beta$-Carotene & & & & & & & & \\
\hline Median $(\mu \mathrm{g})$ & 767.57 & 1473.65 & & 2458.47 & & $4804 \cdot 61$ & & \\
\hline Case/control & $60 / 163$ & $75 / 166$ & & $79 / 166$ & & $70 / 165$ & & \\
\hline Multivariable adjusted* & 1.0 & 1.4 & $0.9,2 \cdot 2$ & $1.7 \dagger$ & $1 \cdot 1,2 \cdot 6$ & 1.6 & $1 \cdot 0,2.5$ & 0.16 \\
\hline Vitamin A & & & & & & & & \\
\hline Median $(\mu \mathrm{g})$ & $160 \cdot 20$ & 291.31 & & 435.24 & & $710 \cdot 76$ & & \\
\hline Case/control & $77 / 165$ & $61 / 165$ & & $77 / 165$ & & $69 / 165$ & & \\
\hline Multivariable adjusted* & 1.0 & 0.7 & $0.5,1 \cdot 2$ & 1.1 & $0.7,1 \cdot 7$ & 1.0 & $0.6,1 \cdot 7$ & 0.67 \\
\hline Vitamin C & & & & & & & & \\
\hline Median (mg) & $25 \cdot 83$ & $51 \cdot 22$ & & 86.45 & & 138.66 & & \\
\hline Case/control & $72 / 168$ & $68 / 164$ & & $73 / 162$ & & $71 / 166$ & & \\
\hline Multivariable adjusted* & 1.0 & $1 \cdot 1$ & $0.7,1 \cdot 7$ & 1.3 & $0.8,1.9$ & 1.2 & $0 \cdot 7,1 \cdot 8$ & 0.53 \\
\hline
\end{tabular}


Table 3. Continued

\begin{tabular}{|c|c|c|c|c|c|c|c|c|}
\hline & \multirow[b]{2}{*}{ Reference } & \multicolumn{2}{|c|}{ Quartile 2} & \multicolumn{2}{|c|}{ Quartile 3} & \multicolumn{2}{|c|}{ Quartile 4} & \multirow[b]{2}{*}{$P$-trend } \\
\hline & & OR & $95 \% \mathrm{Cl}$ & OR & $95 \% \mathrm{Cl}$ & OR & $95 \% \mathrm{Cl}$ & \\
\hline \multicolumn{9}{|l|}{ Vitamin D } \\
\hline Median $(\mu \mathrm{g})$ & 1.52 & $2 \cdot 98$ & & $4 \cdot 61$ & & 8.49 & & \\
\hline Case/control & $81 / 163$ & $64 / 164$ & & $67 / 168$ & & $72 / 165$ & & \\
\hline Multivariable adjusted ${ }^{*}$ & $1 \cdot 0$ & 0.8 & $0.5,1.2$ & 0.8 & $0.5,1 \cdot 3$ & 0.9 & $0.6,1.5$ & 0.99 \\
\hline \multicolumn{9}{|l|}{ Vitamin E } \\
\hline Median (mg) & 3.96 & 6.00 & & 8.28 & & $12 \cdot 13$ & & \\
\hline Case/control & $79 / 163$ & $69 / 168$ & & $62 / 163$ & & $74 / 166$ & & \\
\hline Multivariable adjusted ${ }^{*}$ & 1.0 & 0.9 & $0.6,1.5$ & 0.8 & $0.5,1.3$ & $1 \cdot 1$ & $0 \cdot 6,2 \cdot 0$ & 0.64 \\
\hline \multicolumn{9}{|l|}{ Vitamin $\mathrm{B}_{6}$} \\
\hline Median (mg) & 0.83 & 1.23 & & 1.61 & & $2 \cdot 27$ & & \\
\hline Case/control & $73 / 162$ & $68 / 168$ & & $77 / 162$ & & $66 / 168$ & & \\
\hline Multivariable adjusted $^{\star}$ & $1 \cdot 0$ & $1 \cdot 0$ & $0.6,1.5$ & $1 \cdot 2$ & $0.7,2 \cdot 0$ & $1 \cdot 1$ & $0 \cdot 6,2 \cdot 1$ & 0.66 \\
\hline \multicolumn{9}{|l|}{ Vitamin $B_{12}$} \\
\hline Median $(\mu \mathrm{g})$ & $2 \cdot 66$ & $4 \cdot 26$ & & 5.85 & & $9 \cdot 10$ & & \\
\hline Case/control & $77 / 171$ & $51 / 160$ & & $89 / 164$ & & $67 / 165$ & & \\
\hline Multivariable adjusted ${ }^{*}$ & & 0.8 & $0.5,1.2$ & 1.4 & $0.9,2 \cdot 2$ & 1.0 & $0.6,1.8$ & 0.53 \\
\hline \multicolumn{9}{|l|}{ Thiamin (vitamin $\mathrm{B}_{1}$ ) } \\
\hline Median (mg) & 0.69 & 1.05 & & 1.36 & & 1.97 & & \\
\hline Case/control & $70 / 145$ & $78 / 175$ & & $65 / 168$ & & $71 / 172$ & & \\
\hline Multivariable adjusted ${ }^{*}$ & 1.0 & $1 \cdot 0$ & $0.6,1.7$ & 0.9 & $0.5,1.5$ & $1 \cdot 0$ & $0.5,2.0$ & 0.88 \\
\hline \multicolumn{9}{|l|}{ Riboflavin (vitamin $B_{2}$ ) } \\
\hline Median (mg) & 1.03 & 1.52 & & 2.05 & & 2.90 & & \\
\hline Case/control & $87 / 162$ & $59 / 171$ & & $64 / 146$ & & $74 / 181$ & & \\
\hline Multivariable adjusted ${ }^{*}$ & 1.0 & $0.6 \dagger$ & $0.4,0.9$ & 0.8 & $0.5,1.3$ & 0.7 & $0.4,1.3$ & 0.68 \\
\hline \multicolumn{9}{|l|}{ Niacin } \\
\hline Median (mg) & 9.56 & 13.86 & & 17.92 & & $25 \cdot 72$ & & \\
\hline Case/control & $85 / 167$ & $55 / 162$ & & $75 / 165$ & & $69 / 166$ & & \\
\hline Multivariable adjusted & 1.0 & 0.7 & $0.4,1.1$ & 1.0 & $0.6,1.6$ & 1.0 & $0.5,1.9$ & 0.72 \\
\hline \multicolumn{9}{|l|}{$\mathrm{Ca}$} \\
\hline Median (mg) & 379.97 & 647.65 & & $927 \cdot 15$ & & $1451 \cdot 60$ & & \\
\hline Case/control & $87 / 165$ & $60 / 165$ & & $75 / 165$ & & $62 / 165$ & & \\
\hline Multivariable adjusted ${ }^{\star}$ & 1.0 & 0.7 & $0.4,1.0$ & 0.8 & $0.5,1.3$ & 0.7 & $0.4,1 \cdot 2$ & 0.41 \\
\hline \multicolumn{9}{|l|}{$\mathrm{Fe}$} \\
\hline Median (mg) & 6.26 & 9.56 & & $12 \cdot 31$ & & 18.08 & & \\
\hline Case/control & $83 / 165$ & $64 / 169$ & & $74 / 160$ & & $63 / 166$ & & \\
\hline Multivariable adjusted ${ }^{\star}$ & 1.0 & 0.7 & $0.5,1.2$ & 0.9 & $0.5,1.5$ & 0.8 & $0.4,1.5$ & 0.64 \\
\hline
\end{tabular}

* Models adjusted for matching factors (age and year of enrolment), total energy, income, BMI, smoking and alcohol consumption.

$+P$-value for individual quartile $<0.05$.

endometriosis with 'intermediate' and 'high' servings/week of oil and the Belgian study reported no association of endometriosis risk with margarine and a marginally increased endometriosis risk associated with butter in unadjusted analyses ${ }^{(12,13)}$. We found a decreased risk of disease with increased total fat consumption and similar associations with saturated-, monounsaturated- and transfat consumption. Evaluating the type of fat used during cooking and at the table, we report a decreased disease risk with butter, shortening or margarine use.

The only available information on diet and endometriosis in a US population is a recent report from a large prospective cohort study, focussing on dietary fat intake. In that study, using data from the Nurses' Health Study II, 1199 subjects without infertility self-reported a laparoscopically confirmed diagnosis of endometriosis between 1991 and 2001, and their dietary intake was assessed via a 130-item FFQ at three time points during the follow-up. The total fat intake was not associated with endometriosis risk; however, the highest quintile of trans-fat intake was associated with an increased risk of endometriosis, while the highest quintile of long-chain $n-3$ fatty acid was inversely associated with endometriosis risk. The present results are not consistent with those of Missmer et al. ${ }^{(14)}$; rather we found a decreased risk of endometriosis with some quartiles of total fat, monounsaturated fat and trans-fat consumption using an energy-substitution model. Using the carbohydrate-substitution model similar to Missmer et $a l^{(14)}$, the OR for our estimates and corresponding $95 \%$ CI did not substantially change (results not shown). Furthermore, the present study used medical records to determine a surgically confirmed diagnosis of definite or probable endometriotic disease, while the Nurses' Health Study relied on self-report of laparoscopically confirmed endometriosis, a case definition that includes a more heterogeneous distribution of disease severity and potentially a number of non-cases. Unlike the present study, the Nurses' Health Study did not have information from cases' medical records and therefore could not evaluate the severity other than stratifying on self-reported fertility status (fertile $v$. infertile). Finally, we evaluated nutrients as well as a wide variety of food groups providing 
Table 4. Endometriosis and selected food groups among 284 cases and 660 controls

(Odds ratios and $95 \%$ confidence intervals)

\begin{tabular}{|c|c|c|c|c|c|c|}
\hline & OR & OR & $95 \% \mathrm{Cl}$ & OR & $95 \% \mathrm{Cl}$ & $P$-trend \\
\hline \multicolumn{7}{|l|}{ Servings/d } \\
\hline Dairy & $\leq 1$ & $>1-2$ & & $>2$ & & \\
\hline Case/control & $84 / 145$ & $135 / 352$ & & $54 / 130$ & & \\
\hline Multivariable adjusted ${ }^{*}$ & 1.0 & $0.6 \dagger$ & $0.4,0.9$ & 0.7 & $0.4,1.2$ & 0.13 \\
\hline Vegetables & $\leq 1$ & $>1-3$ & & $>3$ & & \\
\hline Case/control & 90/204 & $154 / 358$ & & $40 / 98$ & & \\
\hline Multivariable adjusted ${ }^{*}$ & 1.0 & 1.0 & $0.7,1.5$ & 1.0 & $0.6,1.7$ & 0.89 \\
\hline Fruitł & $\leq 1$ & $>1-2$ & & $>2$ & & \\
\hline Case/control & $110 / 297$ & $85 / 165$ & & $89 / 198$ & & \\
\hline Multivariable adjusted ${ }^{\star}$ & 1.0 & $1 \cdot 1$ & $0.8,1.6$ & $1.5 \dagger$ & $1 \cdot 2,2 \cdot 3$ & 0.04 \\
\hline \multicolumn{7}{|l|}{ Servings/week } \\
\hline Whole grains & $<1$ & $1-4$ & & $>4$ & & \\
\hline Case/control & $99 / 226$ & $97 / 203$ & & $88 / 222$ & & \\
\hline Multivariable adjusted* & 1.0 & 1.0 & $0.7,1.5$ & 0.8 & $0.6,1.2$ & 0.36 \\
\hline Legumes & $<1$ & $1-2$ & & $>2$ & & \\
\hline Case/control & $149 / 316$ & $54 / 141$ & & $80 / 199$ & & \\
\hline Multivariable adjusted* & 1.0 & 0.9 & $0.6,1 \cdot 3$ & 0.9 & $0.6,1.3$ & 0.57 \\
\hline Red meat & $\leq 2$ & $>2-4$ & & $>4$ & & \\
\hline Case/control & $102 / 209$ & $69 / 185$ & & $112 / 260$ & & \\
\hline Multivariable adjusted & 1.0 & 0.7 & $0.5,1 \cdot 1$ & 0.9 & $0.6,1.4$ & 0.74 \\
\hline Poultry & $\leq 1$ & $>1-2$ & & $>2$ & & \\
\hline Case/control & $138 / 330$ & $70 / 165$ & & $76 / 164$ & & \\
\hline Multivariable adjusted* & 1.0 & 1.1 & $0.7,1.5$ & 1.2 & $0.8,1.7$ & 0.42 \\
\hline Fatty fish (includes halibut) & $\leq 1$ & $>1-2$ & & $>2$ & & \\
\hline Case/control & $89 / 218$ & $54 / 109$ & & $128 / 309$ & & \\
\hline Multivariable adjusted* & 1.0 & $1 \cdot 1$ & $0.7,1 \cdot 7$ & 1.0 & $0.7,1.5$ & 0.94 \\
\hline Non-fatty fish & $\leq 1$ & $>1-2$ & & $>2$ & & \\
\hline Case/control & $133 / 277$ & $70 / 195$ & & $65 / 157$ & & \\
\hline Multivariable adjusted* & 1.0 & 0.7 & $0.5,1.0$ & 0.8 & $0.5,1.1$ & 0.11 \\
\hline Seafood & $\leq 0.5$ & $>0.5$ & & & & \\
\hline Case/control & $149 / 321$ & $123 / 318$ & & & & \\
\hline Multivariable adjusted* & $1 \cdot 0$ & 0.8 & $0 \cdot 6,1 \cdot 1$ & & & \\
\hline
\end{tabular}

* Models adjusted for matching factors (age and year of enrolment), total energy, income, BMI, smoking and alcohol consumption. $\dagger P$-value for individual quartile $<0.05$.

$\ddagger$ Excluding fruit juice.

additional information about food consumption patterns of US women and endometriosis risk.

In our food group analyses, vegetable intake was not associated with endometriosis risk; however, increased disease risk was associated with increased fruit consumption. These findings are not consistent with the reduced risk of endometriosis associated with consumption of both green vegetables and fresh fruit reported by Parazzini et al. ${ }^{(12)}$. Our fruit consumption variable does not differentiate between fresh and canned fruit; however, we did find non-significantly elevated endometriosis risk with individual fresh fruit categories (results not shown). Although speculative, our findings could be related to increased pesticide consumption through fruits. Laboratory analyses have demonstrated that pesticides can remain at detectable levels after harvest and processing, and that pesticide residue is found more commonly in domestic fruits than in imported fruits and more commonly in fruits than in vegetables ${ }^{(22,23)}$. In vitro and in vivo studies have shown that certain classes of pesticides may produce oestrogenic effects $^{(24,25)}$. Further, exposure of rats with induced endometriosis to high doses of a pesticide commonly used on orchard fruits exacerbates the existing endometriotic lesions and contributes to its recurrence ${ }^{(26)}$. We also observed an increased endometriosis risk with increased $\beta$-carotene consumption that could be related to the increase that we observed with servings/d of fruit.

Our other food group result of interest was a marginally significant inverse association between dairy intake and endometriosis. While this association has not previously been observed, decreased risk of breast cancer, another hormone-related disease, has been associated with increased dairy consumption ${ }^{(27)}$. One hypothesis for the mechanism of this association relates to the ability of $\mathrm{Ca}$ and vitamin D to down-regulate growth-promoting factors, such as insulin-like growth factor-I and up-regulate negative growth factor regulators, such as transforming growth factor $\beta$. We did not see significantly decreased endometriosis risk associated with increased vitamin D intake; however, we saw a similar association, albeit not statistically significant, with Ca.

Contrary to the data published by Parazzini et al., we did not report an increased risk of endometriosis with increasing servings/week of red meat ${ }^{(12)}$. Parazzini et al. categorised tertiles of red meat consumption as $0-3$ servings/ week (referent), 4-6 servings/week (intermediate), 7 or more servings/week (high) ${ }^{(12)}$. We categorised red meat consumption in servings/week as well; however, our 
categories were 0-2 servings/week (referent), 2-4 servings/week and $>4$ servings/week. Only $15 \%$ of our population reported eating seven or more servings of meat/week compared with $33 \%$ of participants in the Parazzini study. We may not have found an increased risk because our population ate less meat than the Italian population. We re-evaluated the present results using the tertile categorisation that Parazzini et al. used and did not find an increased risk of endometriosis with frequent red meat consumption (results not shown).

There are several strengths to the present study, including its size and population-based design. Cases without surgical confirmation of endometriosis as well as participants who reported prior surgically confirmed endometriosis were excluded. This allowed us to capture the initial surgical diagnosis of endometriosis and to ascertain exposures before the first medical visit for onset of symptoms associated with that diagnosis. In an attempt to enhance the reproducibility of our findings, a well-defined set of criteria was used to evaluate the certainty of diagnosis. In addition, disease features and evidence were evaluated directly from medical records rather than relying on self-report.

The controls, chosen from the same well-enumerated population, had a race, income and educational profile similar to other western Washington state residents ${ }^{(28)}$. We selected controls randomly from reproductive-age female GH enrollees rather than utilising GH gynaecological patients because the latter may introduce bias if dietary exposures are associated with other gynaecological pathology. One consequence of our choice was that some of our controls may have had undiagnosed endometriosis. From previous studies, we calculate that the presence of undiagnosed, symptomatic endometriosis in the control group was likely to have been $>2 \%$, resulting in a very small number of cases being misclassified as controls ${ }^{(16)}$. Partly to address this issue, we limited our case group to women with definite or probable endometriotic disease, and it is unlikely that participants with this extent of disease were included in the control group. To further address the issue, in one subanalysis, we excluded controls with endometriosis-type symptoms. In this analysis, we found no change in our effect estimates, suggesting that the potential presence of undiagnosed cases in our control group had little impact on the results of the analyses we present.

The most apparent limitation in the present study was the retrospective assessment of dietary factors, a problem inherent in all case-control studies of diet and chronic disease that can lead to measurement error if cases' recall of diet is different from that of the control group. Additionally, since diet in the 12 months before interview was assessed in the present study, cases may have reported healthier diets than controls as a result of post-diagnosis dietary changes. We think these biases are unlikely to have affected our findings for two reasons. First, at the time of the Women's Risk of Endometriosis interviews, dietary recommendations for endometriosis prevention were limited. The first reports on soya and environmental contaminants were published in 2001, and dietary recommendations in published books are copyrighted in 2003 and later. Second, the specificity of the association, increased risk of endometriosis with $\beta$-carotene and fruit and not vegetables also argues against differential recall and post-diagnosis dietary changes with respect to fruit and vegetable consumption. We did not account for multiple comparisons in our analyses; we emphasise that our findings may be due to chance and require further investigation.

Changes in dietary patterns over time, limited recall of serving size and omission of certain foods can contribute to measurement error on the FFQ. It has been demonstrated that FFQ often underestimate energy and fat consumption $^{(29)}$. Measurement error may have occurred in the estimation of dietary intake in the present study; however, it is unlikely that there were systematic differences in dietary assessment by study group. Thus, non-differential misclassification of exposure would tend to underestimate any true associations.

Healthy individuals may be more willing to participate in research studies than less healthy individuals; therefore, we may have an incomplete characterisation of the entire range of dietary intake of all potential cases and controls. Because a high and equivalent percentage of cases and controls participated in the present study, this potential limitation, if present, is unlikely to have caused substantial bias. Given that the present study is a case-control study and information on dietary intake was collected after the diagnosis of endometriosis, we cannot rule out that postdiagnosis dietary changes may have resulted in cases reporting healthier diets than the controls, leading to the decreased risk observed for dietary fat intake and the increased risk with fruit intake.

In summary, the present study suggests a possible inverse risk of disease with dietary fat and dairy consumption and an increased risk of endometriosis with $\beta$-carotene and higher servings of fruit/d, but these findings have not been confirmed elsewhere and require further evaluation in a prospective investigation. A more detailed understanding on the impact of dietary components and patterns on endometriosis risk is urgently needed to inform the development of population-based strategies to prevent this serious and prevalent gynaecological disease.

\section{Acknowledgements}

None of the authors has any conflicts of interest. The present research was supported by grants numbered R01 HD33792 and T32 HD052462 from the National Institute of Child Health and Human Services, National Institute of Health. Pilot grant funding from the Center for Ecogenetics and Environmental Health and the Royalty Research Fund 
at the University of Washington also supported this research. The authors and their contributions are as follows: B. T. was involved with the interpretation and analysis of the data and drafting the manuscript. U. P. was involved with the analysis and interpretation of data and revision of the manuscript. A. J. D. was involved with interpretation of the data and revision of the manuscript. D. S. was involved with study design, data acquisition and revision of the manuscript. V. L. H. was the principal investigator; she was involved with study design, data acquisition, data analysis, interpretation of the data and drafting and revision of the manuscript.

\section{References}

1. Eskenazi B \& Warner ML (1997) Epidemiology of endometriosis. Obstet Gynecol Clin North Am 24, 235-258.

2. Bulun SE (2009) Endometriosis. N Engl J Med 360, 268-279.

3. Kitawaki J, Kado N, Ishihara H, et al. (2002) Endometriosis: the pathophysiology as an estrogen-dependent disease. J Steroid Biochem Mol Biol 83, 149-155.

4. Vigano P, Parazzini F, Somigliana E, et al. (2004) Endometriosis: epidemiology and aetiological factors. Best Pract Res Clin Obstet Gynaecol 18, 177-200.

5. Hediger ML, Hartnett HJ \& Louis GM (2005) Association of endometriosis with body size and figure. Fertil Steril $\mathbf{8 4}$, $1366-1374$.

6. Cramer DW, Wilson E, Stillman RJ, et al. (1986) The relation of endometriosis to menstrual characteristics, smoking, and exercise. JAMA 255, 1904-1908.

7. Signorello LB, Harlow BL, Cramer DW, et al. (1997) Epidemiologic determinants of endometriosis: a hospital-based case-control study. Ann Epidemiol 7, 267-741.

8. Darrow SL, Vena JE, Batt RE, et al. (1993) Menstrual cycle characteristics and the risk of endometriosis. Epidemiology 4, 135-142.

9. Kaneda N, Nagata C, Kabuto M, et al. (1997) Fat and fiber intakes in relation to serum estrogen concentration in premenopausal Japanese women. Nutr Cancer 27, 279-283.

10. Armstrong BK, Brown JB, Clarke HT, et al. (1981) Diet and reproductive hormones: a study of vegetarian and nonvegetarian postmenopausal women. I Natl Cancer Inst 67, $761-767$.

11. Longcope C, Gorbach S, Goldin B, et al. (1987) The effect of a low fat diet on estrogen metabolism. J Clin Endocrinol Metab 64, 1246-1250.

12. Parazzini F, Chiaffarino F, Surace M, et al. (2004) Selected food intake and risk of endometriosis. Hum Reprod 19, 1755-1759.

13. Heilier JF, Donnez J, Nackers F, et al. (2007) Environmental and host-associated risk factors in endometriosis and deep endometriotic nodules: a matched case-control study. Environ Res 103, 121-129.

14. Missmer SA, Chavarro JE, Malspeis S, et al. (2010) A prospective study of dietary fat consumption and endometriosis risk. Hum Reprod 25, 1528-1535.

15. Marino JL, Holt VL, Chen C, et al. (2008) Shift work, hCLOCK T3111C polymorphism, and endometriosis risk. Epidemiology 19, 477-484.

16. Holt VL \& Weiss NS (2000) Recommendations for the design of epidemiologic studies of endometriosis. Epidemiology $\mathbf{1 1}$ 654-659.

17. Patterson RE, Kristal AR, Tinker LF, et al. (1999) Measurement characteristics of the Women's Health Initiative food frequency questionnaire. Ann Epidemiol 9, 178-187.

18. Neuhouser ML, Tinker LF, Thomson C, et al. (2006) Development of a glycemic index database for food frequency questionnaires used in epidemiologic studies. J Nutr 136, 1604-1609.

19. Schakel SF, Sievert YA \& Buzzard IM (1988) Sources of data for developing and maintaining a nutrient database. $J \mathrm{Am}$ Diet Assoc 88, 1268-1271.

20. Willett W (1998) Nutritional Epidemiology. New York, NY: Oxford University Press.

21. Olafsdottir AS, Thorsdottir I, Gunnarsdottir I, et al. (2006) Comparison of women's diet assessed by FFQs and 24hour recalls with and without underreporters: associations with biomarkers. Ann Nutr Metab 50, 450-460.

22. Garcia-Reyes JF, Gilbert-Lopez B, Molina-Diaz A, et al. (2008) Determination of pesticide residues in fruit-based soft drinks. Anal Chem 80, 8966-8974.

23. Katz JM \& Winter CK (2009) Comparison of pesticide exposure from consumption of domestic and imported fruits and vegetables. Food Chem Toxicol 47, 335-338.

24. Morinaga H, Yanase T, Nomura M, et al. (2004) A benzimidazole fungicide, benomyl, and its metabolite, carbendazim, induce aromatase activity in a human ovarian granuloselike tumor cell line (KGN). Endocrinology 145, 1860-1869.

25. Rehnberg GL, Cooper RL, Goldman JM, et al. (1989) Serum and testicular testosterone and androgen binding protein profiles following subchronic treatment with carbendazim. Toxicol Appl Pharmacol 101, 55-61.

26. Cummings AM \& Metcalf JL (1995) Effects of estrogen, progesterone, and methoxychlor on surgically induced endometriosis in rats. Fundam Appl Toxicol 27, 287-290.

27. Moorman PG \& Terry PD (2004) Consumption of dairy products and the risk of breast cancer: a review of the literature. Am J Clin Nutr 80, 5-14.

28. Saunders KW \& Stergachis A (2008) Group health cooperative. In Pharmacoepidemiology, pp. 223-239 [BL Strom, editor]. West Sussex: John Wiley \& Sons, Ltd.

29. Caan BJ, Lanza E, Schatzkin A, et al. (1999) Does nutritionist review of a self-administered food frequency questionnaire improve data quality? Public Health Nutr 2, 565-569. 